\title{
EVALUACIÓN DEL EFECTO A LARGO PLAZO DE INTERVENCIONES EDUCATIVAS PARA EL AUTOCUIDADO DE LA DIABETES
}

\section{ASSESSING THE LONG-TERM EFFECT OF EDUCATIONAL INTERVENTIONS FOR SELF-MANAGEMENT OF DIABETES}

\author{
Jose Alfredo Pimentel Jaimes * \\ Olivia Sanhueza Alvarado * \\ Juana Mercedes Gutiérrez Valverde ${ }^{* * *}$ \\ Esther Carlota Gallegos Cabriales ${ }^{* * * *}$
}

\begin{abstract}
RESUMEN
Objetivo: Analizar la evidencia respecto a la evaluación del efecto a largo plazo de intervenciones educativas para el autocuidado de la diabetes. Material y método: Búsqueda de artículos científicos introduciendo las palabras clave en las bases de datos EBSCO, PUBMED y buscador Google Académico. Se seleccionaron y revisaron artículos publicados desde enero 2001 hasta diciembre 2012. Resultados: Fueron seleccionadas y analizadas ocho intervenciones dirigidas a personas con Diabetes Mellitus Tipo 2 (DMT2) de entre 30 y 85 años de edad, con mal control glucémico (HbAlc $>7.4 \%$ ) al inicio de la intervención. En cuanto a los resultados clínicos y bioquímicos después de la intervención, el IMC y la HbAlc se redujeron significativamente a los 6 meses ( $\mathrm{p}<0.001,1$ estudio), 12 meses ( $\mathrm{p}<0.001,3$ estudios), 2 años ( $\mathrm{p}<0.001,2$ estudios), 3 años ( $\mathrm{p}<0.001,1$ estudio), 4 años ( $\mathrm{p}<0.001,1$ estudio) y 5 años ( $\mathrm{p}<0.001,1$ estudio). Se reportó un buen nivel de conocimientos sobre la diabetes a los dos, cuatro y cinco años de seguimiento ( $\mathrm{p}<0.001,3$ estudios). Se mantuvieron los cambios benéficos en el autocuidado a los 6 y 12 meses, dos, tres y cinco años respectivamente ( $<<0.001,5$ estudios), y también se reportaron mejoras en la calidad de vida a los 6 y 12 meses, dos, tres y cinco años $(\mathrm{p}<0.001,7$ estudios). Conclusión: La participación en una intervención DSME basada en el empoderamiento con apoyo permanente en el autocuidado, puede tener un efecto positivo y duradero en las conductas de autocuidado, la calidad de vida, salud metabólica y cardiovascular.
\end{abstract}

Palabras clave: Diabetes mellitus tipo 2, educación, autocuidado, estudios de seguimiento, evaluación de resultados de intervenciones terapéuticas, enfermería.

\begin{abstract}
Objective: To examine the evidence regarding the evaluation of long-term effect of educational interventions for Diabetes Self-care (DSME). Methods: A search of scientific articles was performed by entering key words in the EBSCO databases, data PUBMED and Google Scholar search engine. For this purpose we selected and reviewed

\footnotetext{
*Enfermero. Docente Universidad Autónoma del Carmen, Campeche, México. E-mail: pimentel_picis@hotmail.com

${ }^{* *}$ Enfermera. Profesora Universidad de Concepción. Concepción, Chile. E-mail: osanhue@udec.cl

${ }^{* * *}$ Enfermera. Profesora Universidad Autónoma de Nuevo León. Nuevo León, México. E-mail: juana.gutierrezv@uanl.mx

${ }^{* * *}$ Enfermera. Profesora Universidad Autónoma de Nuevo León. Monterrey, Nuevo León, México: E-mail: esther.gallegosc@uanl.mx
} 
articles published from January 2001 to December 2012. Results: We selected and analyzed eight interventions for people with Type 2 Diabetes Mellitus (T2DM) between 30 and 85 years of age with poor glycemic control $(\mathrm{HbAlc}>7.4 \%)$ at the start of the intervention. As to of clinical and biochemical outcomes after intervention BMI and HbAlc were significantly reduced at 6 months ( $\mathrm{p}<0.001,1$ study), 12 months ( $\mathrm{p}<0.001,3$ studies), 2 years ( $\mathrm{p}<0.001,2$ studies), 3 years ( $\mathrm{p}<0.001,1$ study), 4 years $(\mathrm{p}<0.001,1$ study) and five years $(\mathrm{p}<0.001,1$ study). A good level of knowledge about diabetes at two, four and five year follow-up ( $p<0.001,3$ studies) were reported. Beneficial changes in self-care at 6 and 12 months, two, three and five years respectively $(p<0.001,5$ studies) were maintained, and also reported improvements in quality of life at 6 and 12 months, two, three and five years ( $\mathrm{p}<0.001,7$ studies). Conclusion: Participation in a DSME intervention based on empowerment with ongoing support in self-care, can have a lasting positive effect on self-care behaviors, quality of life, metabolic and cardiovascular health.

Key words: Type 2 diabetes mellitus, education, self-care, follow-up studies, evaluation of results of therapeutic interventions, nursing.

Fecha recepción: 02/08/13. Fecha aceptación: 22/10/14.

\section{INTRODUCCIÓN}

La diabetes mellitus (DM) es una enfermedad metabólica resultante de defectos en la secreción de insulina, acción de la insulina o ambos, que se asocia con una interacción variable de factores genéticos, ambientales, fisiológicos y conductuales (1). Actualmente más de 382 millones de personas en el mundo padecen esta enfermedad y se estima que alcance los 592 millones para el 2035. Alrededor de 5.1 millones de personas de entre $20 \mathrm{y}$ 79 años murieron a causa de la diabetes en el año 2013, representando una defunción cada seis segundos a causa de esta enfermedad (2).

La DM constituye un problema representativo en el panorama epidemiológico mundial, relacionándose con grandes cargas económicas y sociales, tanto para los individuos como para la sociedad (3). Durante el año 2013 se gastaron más de 548 mil millones de dólares en atención médica para la diabetes (2).

En la actualidad existen diversas estrategias internacionales para disminuir la morbi-mortalidad a causa de la DM, especialmente de la diabetes mellitus tipo 2 (DMT2), dado que representa entre el 85 y $95 \%$ de to- dos los casos de diabetes en el mundo. Estas estrategias se encuentran orientadas hacia la prevención primaria y medidas de control ( 1 , $2,4)$ a través de atención médica continua, educación para el autocuidado del paciente y apoyo permanente para prevenir complicaciones agudas y reducir el riesgo de complicaciones crónicas (4).

La Educación para el Autocuidado de la Diabetes (DSME, por sus siglas en inglés) ha experimentado notables cambios, incorporando componentes psicosociales, cognitivos, conductuales, clínicos y tecnológicos, tanto en sus contenidos como en las estrategias de enseñanza-aprendizaje utilizadas (5). En general, la DSME ha sido facilitada por diversos profesionales de la salud, básicamente por enfermeras ${ }^{1}$, médicos, nutriólogos y psicólogos clínicos en conjunto. Recientemente se ha informado sobre los programas que están siendo facilitados de manera independiente por enfermeras especializadas en diabetes (6).

Evidencia considerable ha reportado la efectividad a corto plazo ( $\leq 6$ meses) de las

\footnotetext{
${ }^{1}$ Para fines de esta revisión, al hablar de enfermeras se hace referencia al profesional de ambos sexos que se desempeña en el área de enfermería.
} 
intervenciones DSME como una estrategia de educación y control de la DMT2 (6-10). Incluso un Meta-análisis de 34 ensayos clínicos controlados (8), cuyo propósito fue determinar el efecto de la educación para el autocuidado de la diabetes dirigida por enfermeras en el control glucémico y factores de riesgo cardiovascular, evidenció que las intervenciones enfermeras fueron efectivas en períodos de seguimiento de uno a seis meses.

Por otro lado también, una revisión sistemática con Meta-análisis de intervenciones educativas dirigidas a fortalecer el autocuidado de personas con DMT2 realizada el año 2012 por Steinsbekk et al., destacó en sus resultados que las mejoras logradas en las intervenciones DSME fueron breves en duración (no mayores a 12 meses). Sin embargo de los 21 estudios analizados sólo seis incluyeron un seguimiento de más de 12 meses, lo que significa que la mayoría de los estudios no evaluaron los resultados a largo plazo (6).

Hasta la fecha, se ha prestado menos atención a la investigación de los efectos duraderos de las intervenciones educativas en personas con DMT2 para verificar las implicancias en su salud (11-13). Por tal motivo, el propósito de esta revisión es analizar la evidencia científica respecto a la evaluación del efecto a largo plazo de intervenciones DSME.

\section{MATERIAL Y MÉTODO}

Se realizó una revisión (14) de artículos científicos acerca de la evaluación del efecto a largo plazo de intervenciones educativas para el autocuidado de la DMT2 en las bases de datos EBSCO, PUBMED y el buscador Google Académico. Para tal propósito se seleccionaron y revisaron los artículos publicados desde enero de 2001 hasta diciembre de 2012. Se utilizaron las siguientes palabras clave en español y su correspondiente en inglés: diabetes mellitus tipo 2, educación, autocuidado, estudios de seguimiento, evaluación de resultados de intervenciones terapéuticas y enfermería.

Una vez identificadas las palabras clave se procedió a realizar la búsqueda electrónica en las bases de datos y el buscador antes mencionados, y se limitó la búsqueda mediante el uso de operadores lógicos o boleanos e ingresando los años establecidos para esta revisión; cabe destacar también que se efectuó la búsqueda manual de artículos a través de las listas de referencias.

Para esto se incluyeron los estudios de intervención que cumplían con los siguientes criterios:

a) Intervenciones dirigidas a personas mayores de 20 años de edad con diagnóstico confirmado de DMT2.

b) Intervenciones destinadas a mejorar dos o más de las variables clínicas (índice de masa corporal [IMC] y presión arterial [PA]), bioquímicas (colesterol total, lipoproteína de alta densidad [HDL], lipoproteína de baja densidad [LDL], triglicéridos y hemoglobina glucosilada [HbAlc]), cognitivas (nivel de conocimientos sobre la DMT2), conductuales (actividades de autocuidado) y psicosociales (calidad de vida y empoderamiento) en los pacientes con DMT2.

c) Intervenciones con periodos de seguimiento y evaluación de más de 12 meses con o sin intervención de refuerzo.

La búsqueda electrónica inicial arrojó un total de 49.900 artículos de investigación, se limitó la búsqueda en la barra de herramientas de búsqueda básica y avanzada, y se obtuvieron un total de 2.015 artículos de los cuales fueron excluidos 1.986 por no cumplir con los criterios de inclusión y resultar como citas duplicadas, resultando un total de 29 estudios para esta forma de búsqueda. De la búsqueda manual de las listas de referencias se identificaron 23 artículos y 17 fueron excluidos por no contar con periodos de seguimiento y evaluación de más de 12 
meses, quedando un total de 6 estudios. En total 35 artículos de la búsqueda electrónica y manual fueron analizados con más detalle, de los cuales se eliminaron 27, puesto que no consideraron las variables psicosociales y para las variables clínicas solo incluyeron los valores de glucosa en ayunas, y por último se eligieron ocho artículos relacionados con el tema central.

A partir del conjunto de artículos que evaluaron el efecto a largo plazo de las intervenciones DSME, se identificó en una fase inicial el autor, país, año de publicación, la población, raza o etnia a la que se dirigió la intervención y el objetivo de ésta y posteriormente para evaluar la calidad metodológica y validez de los mismos se utilizaron las Guías CASPe de lectura crítica y ensayos clínicos (disponibles en http://redcaspe.org/ drupal/?q=node/29), ambas guías permiten cotejar los artículos seleccionados mediante una serie de 11 preguntas estructuradas y tomar una decisión responsable en cuanto a la mejor evidencia disponible. Cabe destacar que la evaluación de los estudios seleccionados se llevó a cabo por dos expertos con diferente formación metodológica y experiencia clínica, uno experto en metodología y estadística y otro experto en contenido de la materia. Después de haber revisado y cribado de manera independiente cada uno de los estudios incluidos ambos expertos determinaron que los estudios eran válidos y fiables para la inclusión en esta revisión.

\section{RESULTADOS}

De los ocho estudios seleccionados, todos correspondieron a ensayos clínicos controlados con poblaciones de 42 hasta 437 individuos. Cinco investigaciones contaron con un grupo experimental y uno de control. La población participante en los estudios estuvo conformada por personas con DMT2 Europeos y Afroamericanos de entre 30 y 85 años de edad, con mal control glucémico al inicio de la intervención (HbAlc $>7.4 \%$ ), con un tiempo de evolución de la enfermedad de menos de un año hasta 45 años.

Las intervenciones realizadas se entregaron en tiempos desde una sola sesión de seis horas hasta 88 sesiones en 24 meses. Los estudios con o sin intervenciones de refuerzo efectuaron mediciones repetidas desde los seis meses hasta los cinco años. Uno de los Meta-análisis (6) citados en este documento describe que cinco estudios informaron haber empleado un modelo teórico en el desarrollo de su intervención, no obstante, éstos mostraron un menor efecto de la intervención en el grupo control. Esto indica que no es necesario un modelo teórico que sustente el programa o intervención para lograr mejores resultados. Aunque puede ser que otros estudios hayan utilizado un modelo teórico, pero no informan de ello, todavía existe la interrogante sobre la utilidad de este tipo de modelos o teorías.

En contraste, se destaca que de las ocho intervenciones incluidas en esta revisión tres $(9,13,15)$ se rigieron bajo la filosofía del empoderamiento de Anderson y Funnell (16); tres $(11,17,18)$ informaron el empleo del modelo ROMEO (Rethink Organization to Improve Education and Outcomes); y las otras dos $(19,20)$ no reportaron el uso de ningún modelo, teoría o filosofía en el desarrollo e implementación de la intervención (Tabla 1).

En síntesis, se analizaron intervenciones DSME ejecutadas en poblaciones y contextos diferentes, desarrolladas y dirigidas principalmente por médicos, enfermeras/os, nutriólogos y psicólogos clínicos en conjunto. El objetivo general de las intervenciones fue mejorar los resultados clínicos, bioquímicos, cognitivos, conductuales y psicosociales de las personas con DMT2. En este mismo orden serán presentados los resultados, tomando en cuenta el orden cronológico de las mediciones efectuadas en las investigaciones analizadas. 


\begin{tabular}{|c|c|c|c|c|c|c|c|c|}
\hline 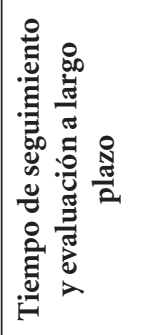 & 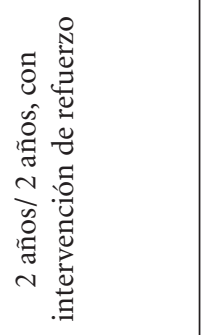 & 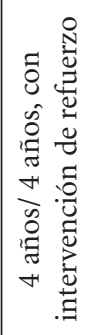 & 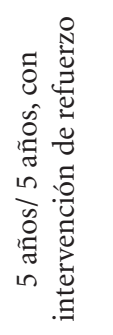 & \multicolumn{2}{|l|}{ 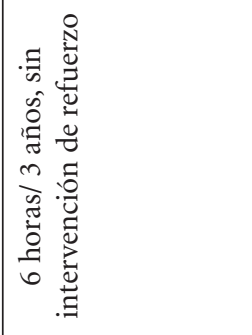 } & 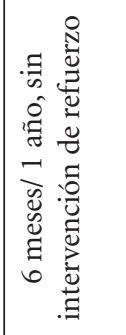 & 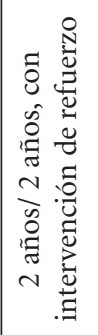 & 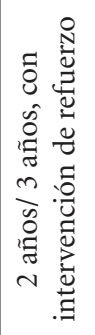 \\
\hline 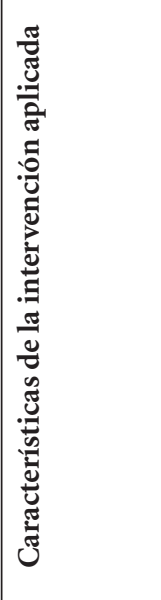 & 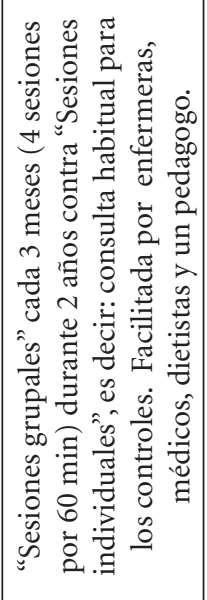 & \multicolumn{2}{|c|}{ 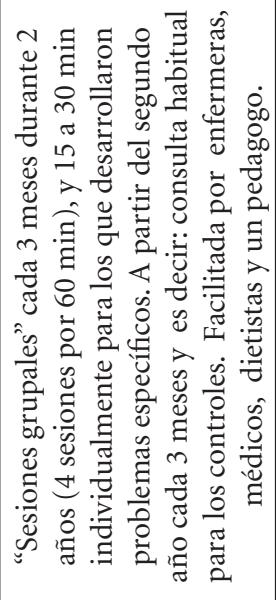 } & \multicolumn{2}{|c|}{ 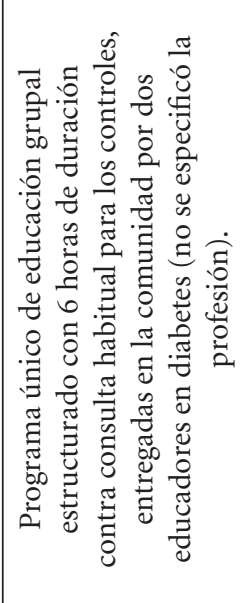 } & 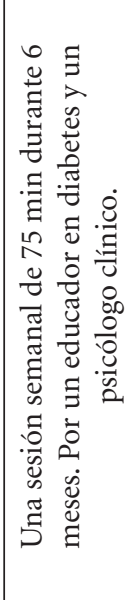 & \multicolumn{2}{|c|}{ 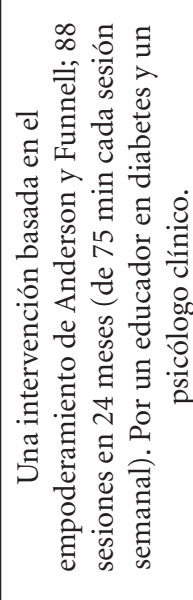 } \\
\hline 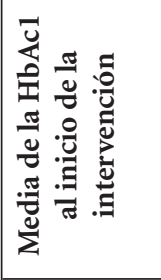 & 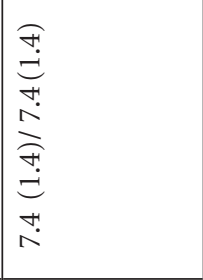 & 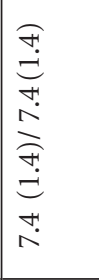 & 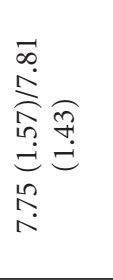 & 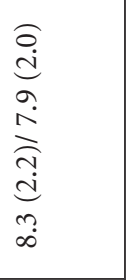 & 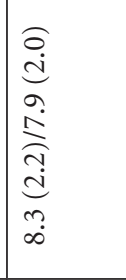 & $\begin{array}{l}\widehat{\overparen{d}} \\
\stackrel{d}{r}\end{array}$ & $\underset{\infty}{\underset{\infty}{d}}$ & 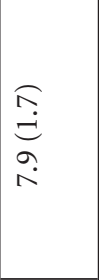 \\
\hline 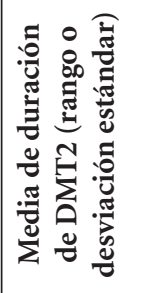 & 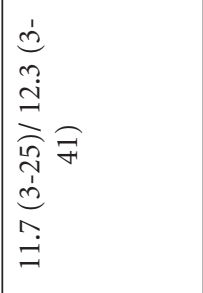 & 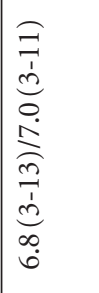 & 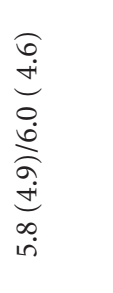 & 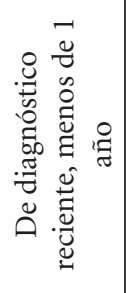 & 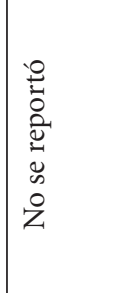 & 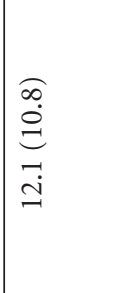 & 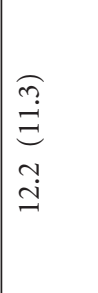 & $\mid \begin{array}{l}\hat{\tilde{n}} \\
\stackrel{\varrho}{\varrho} \\
\stackrel{\infty}{=} \\
=\end{array}$ \\
\hline 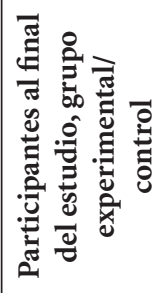 & $\frac{\text { F }}{\stackrel{2}{4}}$ & $\frac{\mathfrak{I}}{\mathfrak{y}}$ & 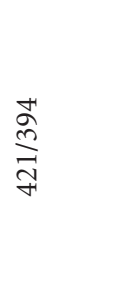 & 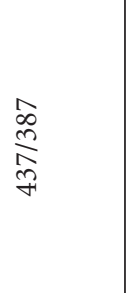 & $\frac{\underset{N}{N}}{\stackrel{N}{n}}$ & 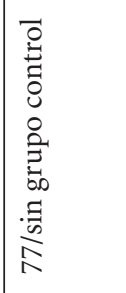 & 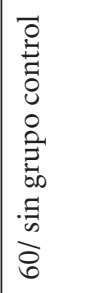 & 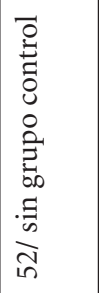 \\
\hline 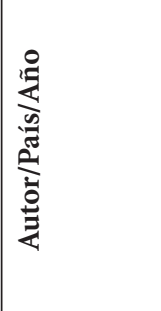 & 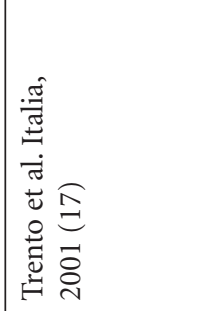 & 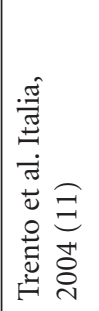 & 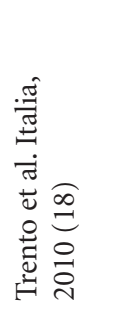 & 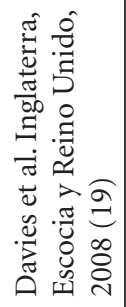 & 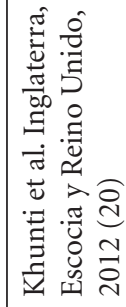 & 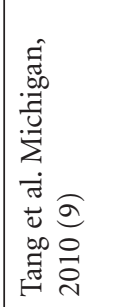 & 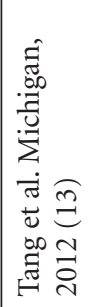 & 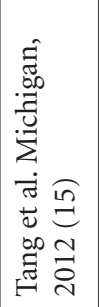 \\
\hline
\end{tabular}




\section{Resultados clínicos y bioquímicos}

En cuanto a los resultados clínicos y bioquímicos, un estudio (9) en una primera medición (seis meses) reportó mejoras significativas en la HbA1c $(\mathrm{p}<0.001)$, el IMC $(\mathrm{p}<0.05)$ y colesterol LDL $(\mathrm{p}<0.001)$ después de la intervención educativa. Tres estudios (9-11) reportaron mejoría del IMC $(\mathrm{p}<0.05)$, colesterol LDL $(\mathrm{p}<0.001)$, PA sistólica/diastólica $(\mathrm{p}<0.05)$ y de la HbA1c $(\mathrm{p}<0.001)$ a los 12 meses, en uno de ellos (11) el colesterol HDL se incrementó.

Para el seguimiento a los dos años se encontraron dos estudios, en uno (18) los niveles de HbAlc y triglicéridos fueron menores en los pacientes atendidos en el grupo experimental que en el grupo control $(\mathrm{p}<0.05)$, y otro (13) reportó además mejoras significativas en la HbAlc $(\mathrm{p}<0.001)$, en el IMC $(\mathrm{p}<0.05)$ y el colesterol LDL $(\mathrm{p}<0.001)$. Sólo dos estudios $(15,19)$ evaluaron los efectos de una intervención a los tres años, en el primero (15) los niveles de HbA1c habían disminuido $(\mathrm{p}<0.001)$, también en el segundo (19) en ambos grupos, sin embargo según los autores después del ajuste estadístico multivariado y el conjunto de la diferencia no fue significativa ( $\mathrm{p}>0.05)$. Un estudio (18) señaló que después de cuatro años de seguimiento de la intervención educativa, el grupo experimental presentó niveles de HbAlc inferiores $(\mathrm{p}<0.001)$. Además se reportó un perfil de lípidos, presión arterial, IMC y creatinina sérica más elevados $(\mathrm{p}<0.001)$ en el grupo control.

Para los resultados clínicos y bioquímicos de una intervención DSME (11) el nivel de HbAlc aumentó progresivamente durante los cinco años entre los participantes del grupo control $(1.7 \%$, IC $95 \% 1.1,2.2)$ pero no en los del grupo experimental $(0.1 \%, 0.5$, $0.4)$, en los cuales disminuyó el IMC (1.4\%, $2.0,0.7)$ y el colesterol HDL se incrementó $(0.14 \% \mathrm{mmol} / 1.0,0.7,0.22)$.

\section{Resultados cognitivos}

El conocimiento sobre la diabetes se evaluó en tres investigaciones $(11,17,18)$ a los dos, cuatro y cinco años, en las cuales se reportó un buen nivel de conocimientos sobre la enfermedad $(\mathrm{p}<0.001)$ después de los refuerzos permanentes de la intervención en los participantes del grupo experimental.

\section{Resultados conductuales}

Para el autocuidado, después de efectuadas las intervenciones $(9,11,13,15,17)$ se presentaron efectos positivos sobre las acciones de autocuidado en los pacientes a los seis meses, 12 meses, dos y cinco años respectivamente; estos efectos se mantuvieron a largo plazo en el grupo experimental $(\mathrm{p}<0.05)$. En los dos estudios restantes las actividades de autocuidado habían disminuido progresivamente tanto en el grupo experimental como el de control $(19,20)$.

\section{Resultados psicosociales}

Respecto a la calidad de vida siete estudios con mediciones repetidas desde los seis meses hasta los cinco años $(9,11,13,15,17,18)$ reportaron mejoras en la calidad de vida para el grupo experimental $(\mathrm{p}<0.001)$. Sólo un estudio (19) no reportó resultados de esta variable, y finalmente el empoderamiento que fue medido en tres estudios $(9,13,15)$ resultó con cambios benéficos en los participantes de la intervención.

\section{DISCUSIÓN Y CONCLUSIÓN}

En la literatura científica se ha reportado ampliamente las características clínicas o los factores que pueden condicionar los resultados clínicos, bioquímicos, cognitivos, conductuales y psicosociales de las personas con DMT2 
(1, 4, 21-24). También se puede notar que los resultados clínicos y bioquímicos son los indicadores estándar de buena salud, dejando de lado la crucial importancia que juegan las variables cognitivas, conductuales y psicosociales para el logro de las primeras $(19,20)$. Tener en cuenta los factores condicionantes (25) a la hora de la elaboración y ejecución de estrategias para las enfermedades crónicas puede resultar tan importante como intentar mantener los parámetros establecidos como normales en el IMC y la HbAlc.

Aunque en los estudios analizados en esta revisión $(9,11,13,15,17-19)$ no se reportan claramente los principales factores que fortalecen, debilitan o intervienen en las actividades de autocuidado de las personas con DMT2 en el largo plazo un estudio reciente (26) realizado por enfermeros/as identificó que son principalmente: el sexo, la edad, limitaciones físicas (de locomoción), problemas de la vista, problemas económicos, el nivel educativo, el trabajo y/o falta de tiempo, "desidia o dejadez", falta de comprensión a las indicaciones de los médicos y la disponibilidad de los mismos.

Tomando en cuenta lo anterior y como profesionales de enfermería, el papel que se asigna a las enfermeras para que consigan que los pacientes puedan llevar a cabo con éxito una estrategia personal de autocuidado es el rol de "entrenador" para que éstos logren ser el gestor eficaz de sus recursos o, en su defecto, asumir la enfermera, por delegación del paciente, este papel, en tanto que el paciente no disponga de la fuerza, el conocimiento o la voluntad $(27,28)$.

Cuando se plantea cómo las enfermeras pueden ayudar a los pacientes y por qué este profesional tiene tanta importancia en el desarrollo de las estrategias de cuidado (29), es porque cuando se habla de autocuidado parece que se trata de algo simple que cualquiera puede desarrollar; al fin y al cabo, el cuidado no es otra cosa que acciones para el mantenimiento de la vida (30). Sin embargo, cuando se razona y se observa la complejidad que involucra un problema de salud como la diabetes y la necesidad de tomar acciones de autocuidado bien ordenadas, sistemáticas y adaptadas (31), es cuando se debe plantear que aunque las acciones de cuidado por definición deben ser sencillas, su secuencia y ordenación en el marco de la cotidianidad deben ser valoradas, estudiadas y planificadas de la mano de un profesional experto en el cuidado (32).

La evidencia científica referente a la evaluación del efecto a largo plazo de las intervenciones DSME permite concluir que los adultos con DMT2 pueden adquirir conocimientos específicos y comportamientos conscientes si se exponen a sesiones educativas grupales con procedimientos y entornos educativos sistematizados adaptados a sus necesidades y características. Además, los resultados sugieren que la participación en una intervención DSME basada en el empoderamiento de Anderson y Funnell (16) con apoyo permanente en el autocuidado de la persona con diabetes, puede tener un efecto positivo y duradero en la conducta de autocuidado, en la calidad de vida, la salud metabólica y cardiovascular de las personas con DMT2.

No obstante, a pesar de los notables cambios que la DSME ha experimentado incorporando componentes psicosociales, cognitivos, conductuales, clínicos y tecnológicos, tanto en sus contenidos como en las estrategias de enseñanza-aprendizaje utilizadas, sigue siendo escasa la investigación que reporte los efectos a largo plazo de estas intervenciones, que pueda apoyar a la identificación de intervenciones educativas efectivas, repetibles y rentables para mantener la salud y retrasar las complicaciones agudas y crónicas de la DMT2.

Los autores recomiendan a los profesionales de la salud, en especial a las/os enfermeras/os, aplicar los resultados de esta revisión, en lo que respecta a la utilización de 
intervenciones DSME que han probado su efectividad a largo plazo en el control de la DMT2, sobre todo las basadas en el empoderamiento, no solo como intervenciones aisladas y únicas, sino que se apliquen refuerzos consecutivos y que se evalúen los efectos constantemente en el tiempo a través de la investigación de enfermería.

La educación es un medio eficaz cuando se combina con el apoyo en el autocuidado grupal e individual mediante programas que dan lugar a cambios positivos y duraderos. Las/os enfermeras/os pueden ser muy eficaces si se centran en el desarrollo de las habilidades de sus pacientes tales como: la fijación de objetivos, la resolución de problemas y gestión de las barreras cognitivas, conductuales y psicosociales como componentes principales del empoderamiento, el cual se ha definido como: "Filosofía que reconoce el derecho fundamental de las personas con diabetes a ser los primeros en la toma de decisiones para el control de su enfermedad. Representa un modelo más compatible del cuidado y la educación necesarios para una enfermedad autocontrolada como es la diabetes".

La esencia del empoderamiento se apoya en tres puntos importantes: enseñar, educar y apoyar, inspirando al paciente. Esta filosofía establece que una persona con diabetes es totalmente responsable de su cuidado, es decir, el equipo de salud no es responsable de las decisiones que tome la persona, pero sí responsable de darle todas las herramientas para que tomen las mejores decisiones y apoyarlo en ellas.

Se ha mencionado también que el papel de la enfermera es fundamental para contener y controlar la epidemia de las enfermedades crónicas, especialmente la DMT2 que es la de mayor prevalencia en el contexto internacional. Pues, se considera a las/os enfermeras/os "como los profesionales expertos en las estrategias relacionadas con el cuidado individual, familiar y grupal, y el profesional con mayor cualificación en lo que se refiere a disponer de herramientas específicas sobre metodología persuasiva, educativa y participativa $y$, por tanto, para planear y dirigir las estrategias de autocuidado en los pacientes con enfermedades crónicas".

De las principales limitaciones presentadas en esta revisión, es que además del número reducido de estudios seleccionados y analizados, el tamaño de muestra fue variable y pequeño para las intervenciones basadas en el empoderamiento de Anderson y Funnell en comparación con otros estudios de intervención multicéntricos, los cuales en el seguimiento también incluyeron a un grupo control. Por otra parte, aunque profesionales de enfermería participaron en la ejecución de algunas intervenciones no se encontraron estudios ideados, desarrollados, implementados y evaluados más allá de los 12 meses por estos profesionales.

Dadas las características de los estudios incluidos en la presente revisión, no se pudo observar hasta qué punto es posible atribuir, con un cierto grado de certeza, el cambio observado a la intervención analizada. Es decir, cuáles de las variables pudieran estar mediando o influyendo sobre las mejoras presentadas en los pacientes seguidos en el tiempo, como la asistencia frecuente a la consulta de rutina, información proveniente de los medios de comunicación e información, la implementación de programas específicos para la diabetes, terapias alternativas, entre otras.

\section{REFERENCIAS}

1. American Diabetes Association. Diagnosis and classification of Diabetes Mellitus. Diabetes Care. 2014; 37(Suppl 1): S81-S90.

2. Federación Internacional de Diabetes. Atlas de la diabetes de la FID [Internet]. Federación Internacional de Diabetes; 2013 [citado 22 octubre 2014]. Disponi- 
ble en: http://www.idf.org/sites/default/ files/SP_6E_Atlas_Full.pdf

3. Arredondo A, De Icaza E. Costos de la diabetes en América Latina: Evidencias del caso Mexicano. Value in Health. 2011; 14(Suppl 1): S85-S88.

4. American Diabetes Association. Standards of medical care in diabetes mellitus. Diabetes Care. 2014; 37(Suppl 1): S14-S80.

5. Funnell MM, Brown TL, Childs BP, Haas LB, Hosey GM, Jensen B, et al. National standards for diabetes self-management education. Diabetes Care. 2009; 32 (Suppl 1): S87-S94.

6. Steinsbekk A, Rygg L, Lisulo M, Rise MB, Fretheim A. Group based diabetes self management education compared to routine treatment for people with type 2 diabetes mellitus. A systematic review with meta-analysis. BMC Health Serv Res. 2012; 12(213): 1-19.

7. Cooper H, Booth K, Gill G. A trial of empowerment-based education in type 2 diabetes global rather than glycaemic benefits. Diabetes Res Clin Pract. 2008; 82 (2): 165-171.

8. Tshiananga JK, Kocher S, Weber C, Erny-Albrecht K, Berndt K, Neeser K. The effect of nurse-led diabetes self-management education on glycosylated hemoglobin and cardiovascular risk factors. A meta-analysis. Diabetes Educ. 2012; 38 (1): 108-23.

9. Tang T, Funnell M, Brown M, Kurlander J. Self-management support in "real-world" settings: an empowerment-based intervention. Patient Educ Couns. 2010; 79(2): 178-184.

10. Vargas A, González A, Aguilar M, Moreno Y. Estudio comparativo del impacto de una estrategia educativa sobre el nivel de conocimientos y la calidad de vida en pacientes con diabetes mellitus tipo 2 . Rev Fac Med UNAM. 2010; 53(2): 60-68.

11. Trento M, Passera P, Borgo E, Tomalino M, Bajardi M, Cavallo F, et al. A 5-year randomized controlled study of learning, problem solving ability, and quality of life modifications in people with type 2 diabetes managed by group care. Diabetes Care. 2004; 27(3): 670-675.

12.Hornsten A, Stenlund H, Lundman B, Sandstom H. Improvements in $\mathrm{HbAlc}$ remain after 5 years-a follow up of an educational intervention focusing on patients' personal understandings of type 2 diabetes. Diabet Res Clin Pract. 2008; 81(1): 50-55.

13.Tang T, Funnell M, Noorulla S, Oh M, Brown M. Sustaining short-term improvements over the long-term: results from a 2-year diabetes self-management support (DSMS) intervention. Diabetes Res Clin Pract. 2012; 95(1): 85-92.

14. Russel CL. An overwiew of the integrative research review. Prog Transplant. 2005; 15(1): 8-13.

15. Tang ST, Funnell MM, Oh M. Lasting effects of a 2-year diabetes self-management support intervention: outcomes at 1-year follow-up. Prev Chronic Dis. 2012; 9: E109.

16. Anderson RM, Funnell MM. Patient empowerment: reflections on the challenge of fostering the adoption of a new paradigm. Patient Educ Couns. 2005; 57(7): 153-157.

17. Trento M, Passera P, Tomalino M, Bajardi M, Pomero F, Allione A, et al. Group visits improve metabolic control in type 2 diabetes: a 2-year follow-up. Diabetes Care. 2001; 24(6): 995-1000.

18. Trento M, Gamba S, Gentile L, Grassi G, Miselli V, Morone G, et al. Rethink organization to improve education and outcomes (ROMEO): a multicenter randomized trial of lifestyle intervention by group care to manage type 2 diabetes. Diabetes Care. 2010; 33(4): 745-747.

19. Davies MJ, Heller S, Skinner TC, Campbell MJ, Carey ME, Cradock S, et al. Effectiveness of the diabetes education and self-management for ongoing and newly 
diagnosed (DESMOND) programme for people with newly diagnosed type 2 diabetes: cluster randomised controlled trial. BMJ. 2008; 336(7642): 491-495.

20. Khunti K, Gray LJ, Skinner T, Carey ME, Realf K, Dallosso H, et al. Effectiveness of a diabetes education and self-management programme (DESMOND) for people with newly diagnosed type 2 diabetes mellitus: three year follow-up of a cluster randomised controlled trial in primary care. BMJ. 2012; 344: e2333.

21. Instituto Nacional de Salud Pública. Encuesta Nacional de Salud y Nutrición [Internet]. México: Instituto Nacional de Salud Pública; 2012 [citado 22 oct 2014]. Disponible en: http://ensanut.insp.mx/

22. Khattab M, Khader YS, Al-Khawaldeh A, Ajlouni K. Factors associated with poor glycemic control among patients with Type 2 diabetes. J Diabetes Complications. 2010; 24(2): 84-89.

23. Ardeňa GJ, Paz-Pacheco E, Jimeno CA, Lantion-Ang FL, Paterno E, Juban N. Knowledge, attitudes and practices of persons with type 2 diabetes in a rural community: phase I of the community-based Diabetes Self-Management Education (DSME) Program in San Juan, Batangas, Philippines. Diabetes Res Clin Pract. 2010; 90(2): 160-166.

24. Misra R, Lager J. Predictors of quality of life among adults with type 2 diabetes mellitus. J Diabetes Complications. 2008;
22 (3): 217-223.

25. Orem D. Nursing: Concepts of practice. 6a ed. St. Louis, MO: Mosby; 2001. 542 p. 26. Pimentel-Jaimes JA, Gutiérrez-Valverde JM, Gallegos-Cabriales EC, Vinicio-Gómez M. Evaluación a largo plazo de una intervención educativa en adultos con diabetes mellitus tipo 2. [Tesis Maestría]. México: Universidad Autónoma de Nuevo León; 2014. p. 42-51.

27. García GM. El proceso de enfermería y el modelo de Virginia Henderson. 2a ed. México D.F.: Progreso; 2004.

28. Marriner A, Raile M. Modelos y teorías en enfermería. 6a ed. España: Elsevier; 2008. 850 p.

29. García HM, Cárdenas BL, Arana GB, Monroy RA, Hernández OY, Salvador MC. Construcción emergente del concepto: cuidado profesional de enfermería. Texto contexto- enferm. 2011; 20(Esp): 74-80.

30. Achury MD. La historia del cuidado y su influencia en la concepción y evolución de la enfermería. Investigación en Enfermería: Imagen y Desarrollo. 2006; 8(1-2): 8-15.

31. Fawcett J. Analisis and evaluation of conceptual models of nursing. $2 \mathrm{a}$ ed. Philadelphia: F.A. Davis; 1989. 392 p.

32. Juárez-Rodríguez PA， García-Campos ML. La importancia del cuidado de enfermería. Rev Enferm Inst Mex Seguro Soc. 2009; 17(2): 109-111. 